\title{
A Note on Exact Conditional and Unconditional Tests for Hardy-Weinberg Equilibrium
}

\author{
Guogen Shan
}

Department of Environmental and Occupational Health, Epidemiology and Biostatistics Program, University of Nevada Las Vegas, Las Vegas, Nev., USA

\section{Key Words}

Conditional test · Exact test · Hardy-Weinberg equilibrium · Unconditional test

\begin{abstract}
The exact conditional approach is frequently used for testing Hardy-Weinberg equilibrium in population genetics. This approach respects the test size as compared to the traditionally used asymptotic approaches. It is a full-enumeration method and very computational. Many efficient algorithms have been successfully developed to implement this exact approach. An alternative to the conditional approach is the unconditional approach, which relaxes the restriction of the fixed number of allelic counts as in the conditional approach. The first unconditional test considered in this study is the one based on maximization, which has been shown to be more powerful than the conditional test to loci with two alleles for small sample sizes. By using the $p$ value of the conditional approach as a test statistic in the following maximization step, the second unconditional test is uniformly more powerful than the conditional approach. We compared these exact tests based on three commonly used test statistics with regards to type I error rate and power. It is recommended to use the second unconditional approach in practice due to the power gain in the case with two alleles.
\end{abstract}

(c) 2013 S. Karger AG, Basel

\section{Introduction}

In population genetics, Hardy-Weinberg equilibrium (HWE) is one of the most important questions to be asked in the first place [1-3]. HWE states that both the allele frequencies and the genotype frequencies remain constant in a population. In addition to that, the alleles for the next generation for any given individual are chosen randomly and independent of each other under the assumption of random mating in an infinite population. The departures from HWE could be caused by factors such as selection, non-random mating, etc. [2]. Testing for HWE has been studied extensively for decades [4-7], and many improved algorithms have been proposed to overcome the computational burden [7]. Traditionally, the testing procedure relies on asymptotic approaches using either the Pearson $\chi^{2}$ test or the likelihood ratio (LR) test $[8,9]$. Although the asymptotic approaches are easy to implement, they are often criticized for their unreliable results, for example, inflated type I error rates [10]. The power of a study would be overestimated due to the overestimated type I error rate. These asymptotic approaches may become unreliable for small to medium sample sizes or for alleles with low probability [7].

In view of the unreliable estimates from the asymptotic approaches, procedures based on exact conditional probabilities may be considered in order to guarantee the

\section{KARGER}

E-Mail karger@karger.com

www.karger.com/hhe
(C) 2013 S. Karger AG, Basel

$0001-5652 / 13 / 0761-0010 \$ 38.00 / 0$
Guogen Shan

Department of Environmental and Occupational Health

Epidemiology and Biostatistics Program, University of Nevada Las Vegas

Las Vegas, NV 89154 (USA)

E-Mail guogen.shan@unlv.edu 
test size $[5,6]$. The exact conditional approach (referred to as the $\mathrm{C}$ approach) $[7,10]$ is the most frequently used approach among exact approaches. This approach is based upon the conditional probability of genotype counts with fixed allelic counts from the observed data, and the associated $\mathrm{p}$ value is calculated by enumerating all possible genotype counts. Full-enumeration methods are often criticized for their heavy computational demand for large sample sizes to enumerate all possible genotype counts [7, 11]. Guo and Thompson [11] were among the first to propose efficient algorithms for applying the conditional approach to loci with multiple alleles for use in practice. Recently, Engels [7] has proposed a substantially improved algorithm based on a lattice-like network [12] to implement the exact conditional approach as well as the Monte Carlo algorithm for large sample sizes based on three commonly used test statistics.

An alternative to the exact conditional approach is the exact unconditional approach based on maximization (referred to as the $M$ approach) $[13,14]$. The $p$ value of the $\mathrm{M}$ approach is calculated as the maximum of the tail probability over the whole range of the unknown nuisance parameters. It has been applied to many important statistical and medical problems $[13,15]$ and has been shown to be at least as powerful as the exact conditional approach in different settings [16, 17]. Haber [16] was among the first researchers to introduce the unconditional test based on maximization for testing HWE to loci with two alleles. The unconditional approach generally has more power than the conditional approach in small sample sizes due to the discreteness of the null distribution in the conditional approach. Haber [16] also argued that the restriction of the fixed number of allelic counts in the conditional approach may not be realistic since the number of allelic counts may be considered as random variables and not fixed values. Discussions about conditional and unconditional approaches may be found in Irony and Pereira [18], Pereira and Lindley [19], Maiste and Weir [20], Rohlfs and Weir [21], Brentani et al. [22], Shan and Ma [23], and Shan et al. [24]. Boschloo [25] proposed another unconditional approach based on the $\mathrm{C}$ approach and the $\mathrm{M}$ approach using the $\mathrm{p}$ value of the $\mathrm{C}$ approach as a test statistic in the following maximization step. This unconditional approach is always uniformly more powerful than the $\mathrm{C}$ approach. In this approach, one needs to calculate the conditional $p$ value for each possible genotype count, and this $\mathrm{p}$ value is then used as a test statistic in the following maximization step. As far as we know, very little work has been done for testing HWE by using the unconditional approach. We compare the exact conditional approach

Note on Exact Conditional and Unconditional Tests for HWE and unconditional approaches based on three commonly used test statistics to loci with two alleles with regards to type I error rate and power.

The rest of this article is organized as follows: first, we briefly review the exact conditional and unconditional approaches for testing HWE. Three commonly used test statistics are also introduced. Then, we compare the performance of the competing tests by studying the actual type I error rate and power of the procedures under various configurations. The last section is given to discussion.

\section{Methods}

The commonly used exact approach for testing HWE is the conditional approach, in which the total number of $A$ genes $\left(n_{A}\right)$ is fixed. The actual type I error rate of the test is computed by enumerating all possible array $\mathbf{n}=\left(n_{A A}, n_{A B}, n_{B B}\right)$ with the same marginal $n_{A}$. By fixing the number of $A$ genes $n_{A}$, the probability of each possible array $\mathbf{n}=\left(n_{A A}, n_{A B}, n_{B B}\right)$ does not depend on any nuisance parameter, and the associated probability is given as

$$
P\left(\mathbf{n} \mid n_{A}\right)=P\left(n_{A B} \mid n_{A}\right)=\frac{N ! n_{A} ! n_{B} ! 2^{n_{A B}}}{(2 N) ! n_{A A} ! n_{A B} ! n_{B B} !},
$$

where $N$ is the total number of subjects, and $n_{B}$ is the total number of $B$ genes $\left(n_{B}=2 n_{B B}+n_{A B}\right.$, and $\left.N=n_{A A}+n_{A B}+n_{B B}\right)$. The rejection region of the test is often determined by the test statistic. The most commonly used test statistic is the Pearson $\chi^{2}$ test

$$
T_{\chi^{2}}=\frac{4 N\left(n_{A A} n_{B B}-n_{A B}^{2} / 4\right)^{2}}{n_{A}^{2} n_{B}^{2}},
$$

which asymptotically follows a $\chi^{2}$ distribution with one degree of freedom (d.f.). This asymptotic approach is often utilized for testing HWE. Wigginton et al. [10] showed that this asymptotic approach always has inflated type I error rates even for relatively large sample sizes $(n=1,000)$. They compared the asymptotic approach and the $\mathrm{C}$ approach with regards to the type I error control and recommended the $\mathrm{C}$ approach for the use in practice. For this reason, we do not compare the asymptotic approach with the exact approaches here, and readers may refer to the comparison between them in Wigginton et al. [10].

Another popular test statistic for testing HWE is the LR test [9], which can be expressed as

$$
T_{L R}=\frac{N^{N}}{(2 N)^{2 N}} \frac{2^{n_{A B}} n_{A}^{n_{A}} n_{B}^{n_{B}}}{n_{A A}^{n_{A A}} n_{A B}^{n_{A B}} n_{B B}^{n_{B B}}} .
$$

It is well known that $-2 T_{L R}$ asymptotically follows a $\chi^{2}$ distribution with d.f. $=1$.

The last but not the least test statistic considered here is the probability. For each array $\mathbf{n}^{\#}$ with the total number of $A$ genes $n_{A}^{\#}$, the associated probability, $T_{\text {Probability }}$, is calculated as the probability of all arrays with the same $n_{A}^{\#}$ of which the conditional probability in Equation (1) is less than or equal to that of the given array

$$
T_{\text {Probability }}\left(\mathbf{n}^{\#}\right)=P\left(\mathbf{n} \mid n_{A}^{\#}: P\left(\mathbf{n} \mid n_{A}\right) \leq P\left(\mathbf{n}^{\#} \mid n_{A}^{\#}\right), n_{A}=n_{A}^{\#}\right) .
$$


Fig. 1. Type I error plots for the $\mathrm{C}$ approach, the $\mathrm{M}$ approach, and the $\mathrm{C}+\mathrm{M}$ approach based on the Pearson $\chi^{2}$ test statistic for $N=20,80,200$, and 1,000 in the rows 1 , 2,3 , and 4 , respectively.

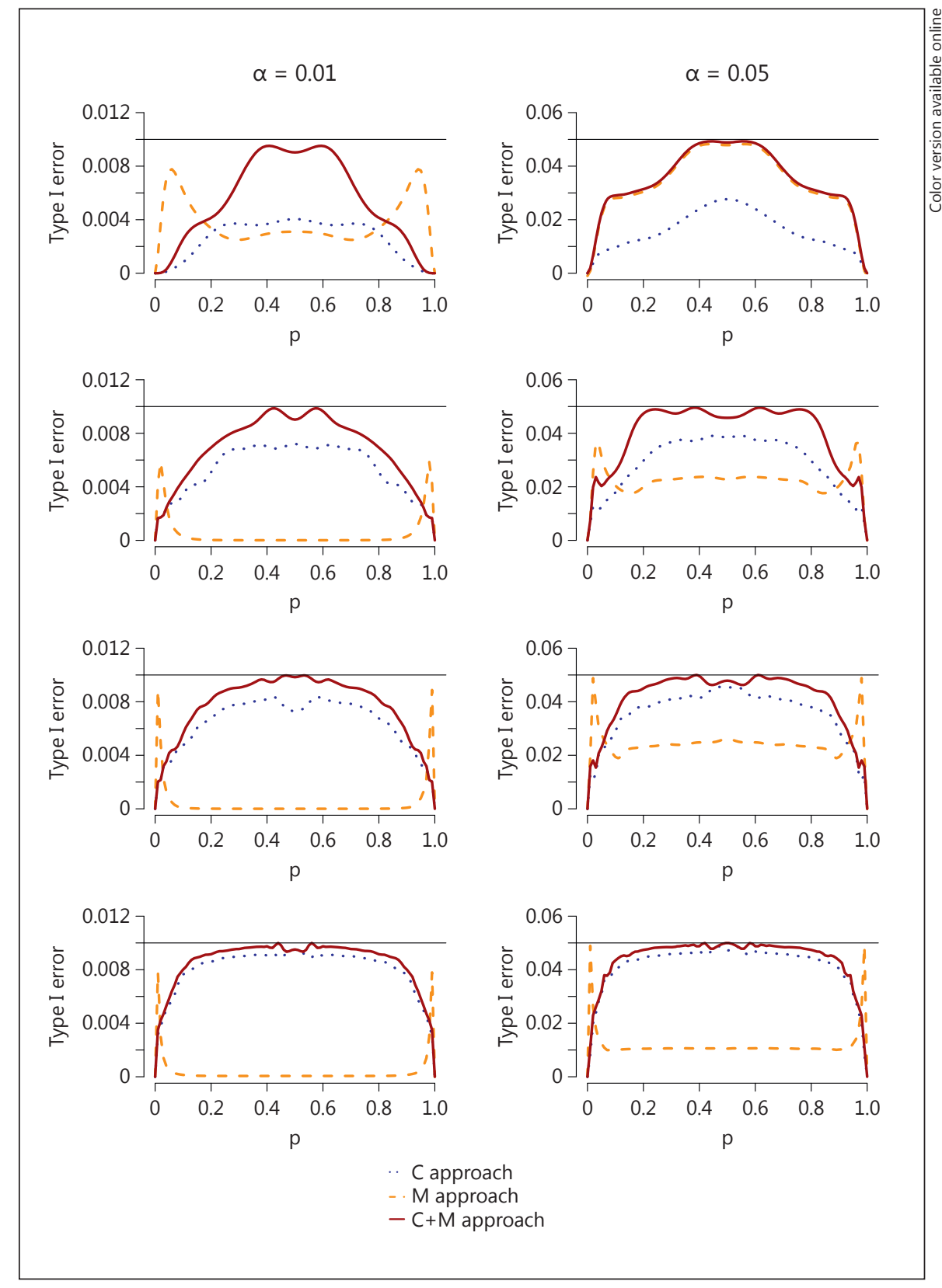

While both $T_{\chi^{2}}$ and $T_{L R}$ reject HWE for large values, the $T_{\text {Probability }}$ reject the null hypothesis for small values. Without further notice, the HWE will be rejected for large values since one can always use $-T_{\text {Probability }}$ as the test statistic to have the same rejection direction as the other two. Although other test statistics may be considered, these three test statistics are the most commonly used for testing HWE. It should be noted that different test statistics may lead to different rejection regions for the given data.

In the $\mathrm{C}$ approach, the conditional null distribution contains all possible array $\mathbf{n}$ given $n_{A}$. It is easy to show that $n_{A B}$ and $n_{A}$ are ei- ther odds or evens since the difference between them is even: $n_{A}-$ $n_{A B}=2 n_{A A}$. Let $\mathbf{n}^{*}=\left(n_{A A}^{*}, n_{A B}^{*}, n_{B B}^{*}\right)$ be an observed array for the data, the conditional $\mathrm{p}$ value based on the test statistic $T$ is given as

$$
P_{C}\left(\mathrm{n}^{*}\right)=P\left(\mathbf{n} \mid n_{A}^{*}: T(\mathbf{n}) \geq T\left(\mathbf{n}^{*}\right), n_{A}=n_{A}^{*}\right) .
$$

The $\mathrm{C}$ approach has been investigated for decades, and many efficient algorithms have been proposed (e.g. see Wigginton et al. [10] and Engels [7]).

An alternative to the exact $\mathrm{C}$ approach is the unconditional approach. As mentioned above, Haber [16] was among the first re- 


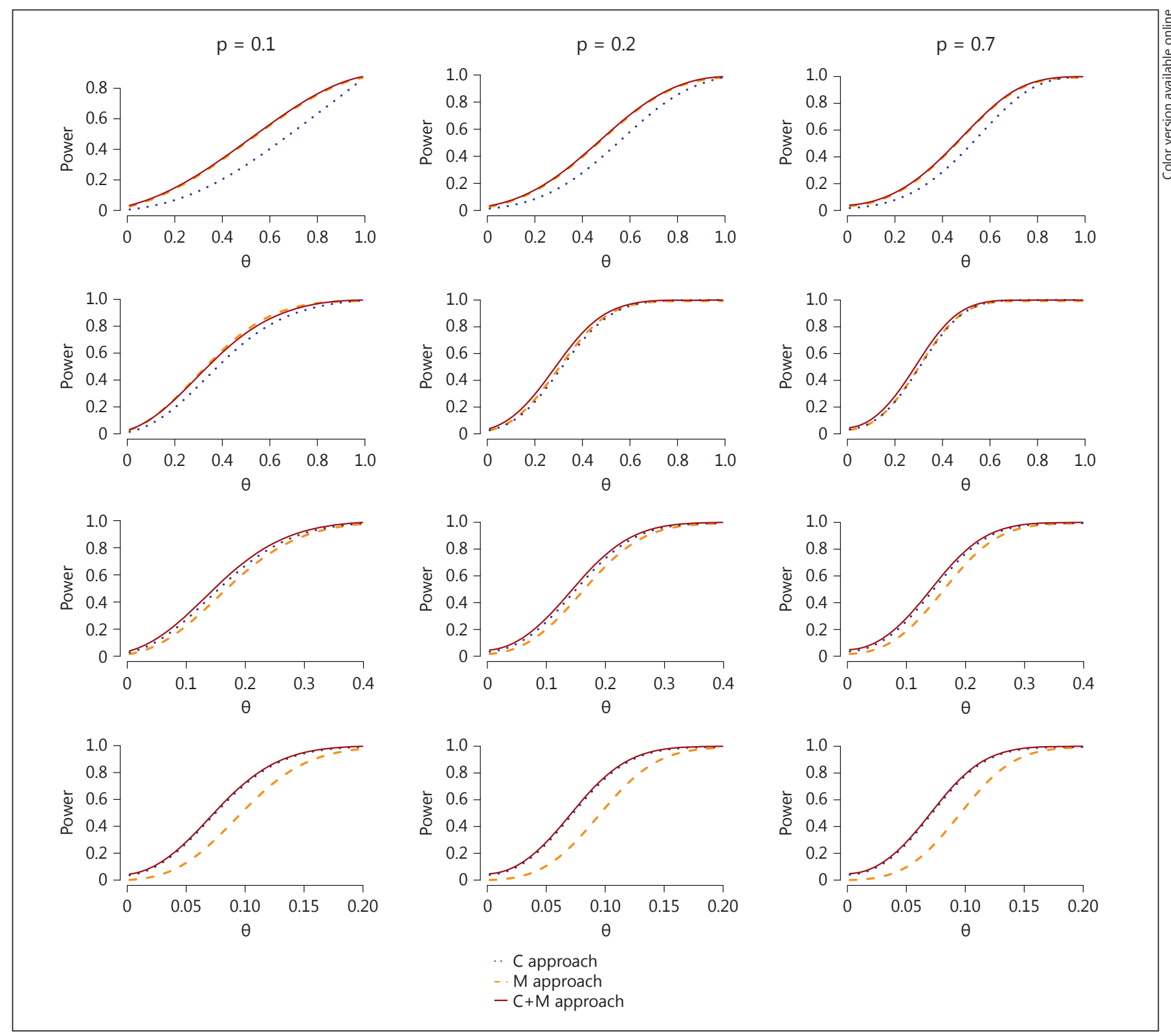

Fig. 2. Power comparison among the $C$ approach, the $M$ approach, and the $C+M$ approach based on the Pearson $\chi^{2}$ test statistic for $N=20,50,200$, and 800 in the rows $1,2,3$, and 4 , respectively.

searchers to introduce the unconditional test for testing HWE. The unconditional distribution of an array $\mathbf{n}$ is multinomial with parameters $\left(N ; p_{A A}, p_{A B}, p_{B B}\right)$, and the associated probability can be expressed as

$$
P\left(\mathbf{n} \mid p_{A A}, p_{A B}, p_{B B}\right)=\frac{N !}{n_{A A} n_{A B} n_{B B}} p_{A A}^{n_{A A}} p_{A B}^{n_{A B}} p_{B B}^{n_{B B}}
$$

Under the assumption of HWE, $H_{0}: p_{A A}=p^{2}, p_{A B}=2 p(1-p)$, $p_{B B}=(1-p)^{2}$, the unconditional null distribution is then given as

Note on Exact Conditional and Unconditional Tests for HWE

$$
P_{0}(\mathbf{n} \mid p)=\frac{N !}{n_{A A} n_{A B} n_{B B}} 2^{n_{A B}} p^{2 n_{A A}+n_{A B}}(1-p)^{2 n_{B B}+n_{A B}},
$$

where $0 \leq p \leq 1$. There is a nuisance parameter, $p$, in the null likelihood $P_{0}(\mathbf{n} \mid p)$. Therefore, the tail probability is a function of $p$, not a numeric value. One way to eliminate this nuisance parameter is to allow for the worst case [14]. The exact unconditional p value using maximization (referred to as the $\mathrm{M} \mathrm{p}$ value) is then defined as the maximum of the surface of the tail probability over the whole range of the nuisance parameter. In this way, the unconditional 


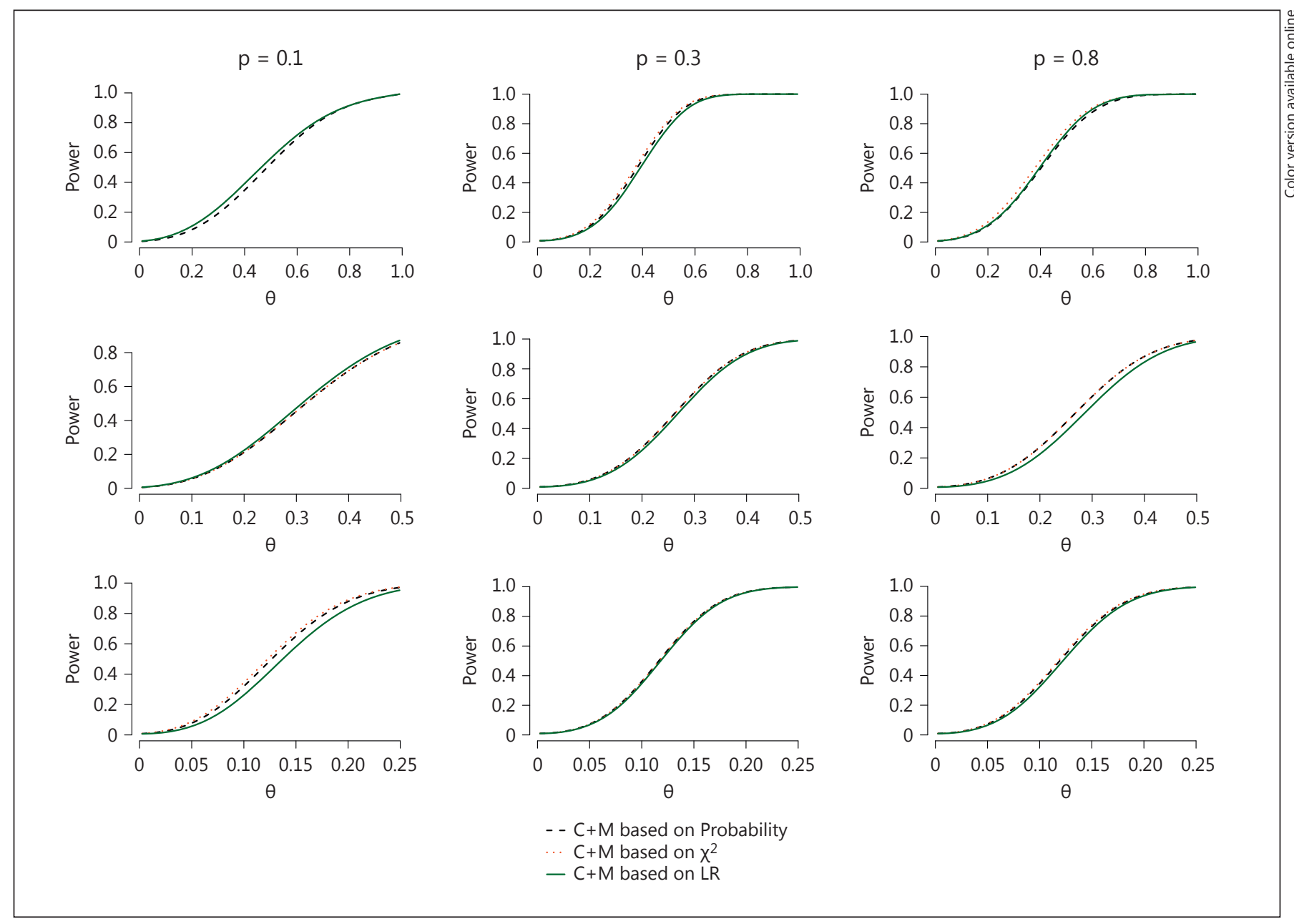

Fig. 3. Power comparison for the $\mathrm{C}+\mathrm{M}$ approach based on the three different test statistics at the nominal level of 0.01 for $N=50,100$, and 500 in the rows 1,2 , and 3, respectively.

approach is able to control for the dependence of the testing procedure on the nuisance parameter. The $\mathrm{M} \mathrm{p}$ value [16] is given as

$$
P_{M}\left(\mathbf{n}^{*}\right)=\sup _{p \in[0,1]}\left[\sum_{\mathbf{n} \in \Omega_{T}\left(\mathbf{n}^{*}\right)} P_{0}(\mathbf{n} \mid p)\right],
$$

where $\Omega_{T}\left(\mathbf{n}^{*}\right)=\left\{\mathbf{n}: T(\mathbf{n}) \geq T\left(\mathbf{n}^{*}\right)\right\}$ is the rejection region. The size of the unconditional null distribution could be much larger than that of the conditional null distribution due to the fixed marginal for the conditional approach. It may result in a very discrete null distribution for the conditional approach for small sample sizes and lead to conservative results when comparing to the unconditional approach. Haber [16] showed that the M approach is usually more powerful than the $\mathrm{C}$ approach based on the $T_{\chi}{ }^{2}$ for small sample sizes up to 100 , and the $M$ approach requires smaller sample sizes to attain a given power as compared to the $\mathrm{C}$ approach.

Boschloo [25] proposed an unconditional approach based on the $\mathrm{C}$ approach and the $\mathrm{M}$ approach, where the conditional $\mathrm{p}$ val- ue is used as a test statistic for the following maximization step. We referred to this approach as the $\mathrm{C}+\mathrm{M}$ approach. The conditional $\mathrm{p}$ value $P_{C}(\mathbf{n})$ in Equation (2) is firstly calculated for each $\mathbf{n}$ in the sample space; the rejection region of the $\mathrm{C}+\mathrm{M}$ approach is then determined as the collection of $\mathbf{n}$ with $\mathrm{p}$ values less than or equal to the conditional $p$ value of the given data. In this testing procedure, the conditional $p$ value is used as a test statistic, not a final $p$ value for the data, which may cause some confusion (for more details about the $\mathrm{C}+\mathrm{M}$ approach, see Boschloo [25]). Using the conditional $p$ value as a test statistic and then maximizing the tail probability over the range of the nuisance parameter, the $\mathrm{C}+\mathrm{M}$ approach may be able to reduce the conservatism of the $\mathrm{C}$ approach in small to medium sample sizes. The rejection region for the $\mathrm{C}+\mathrm{M}$ approach is given as $\Omega_{C}\left(\mathbf{n}^{*}\right)=\left\{\mathbf{n}: P_{C}(\mathbf{n}) \leq P_{C}\left(\mathbf{n}^{*}\right)\right\}$, and the $\mathrm{C}+\mathrm{M}$ $p$ value is

$$
P_{C+M}\left(\mathbf{n}^{*}\right)=\sup _{p \in[0,1]}\left[\sum_{\mathbf{n} \in \Omega_{C}\left(\mathbf{n}^{*}\right)} P_{0}(\mathbf{n} \mid p)\right] .
$$

14

Hum Hered 2013;76:10-17 DOI: $10.1159 / 000353205$ 


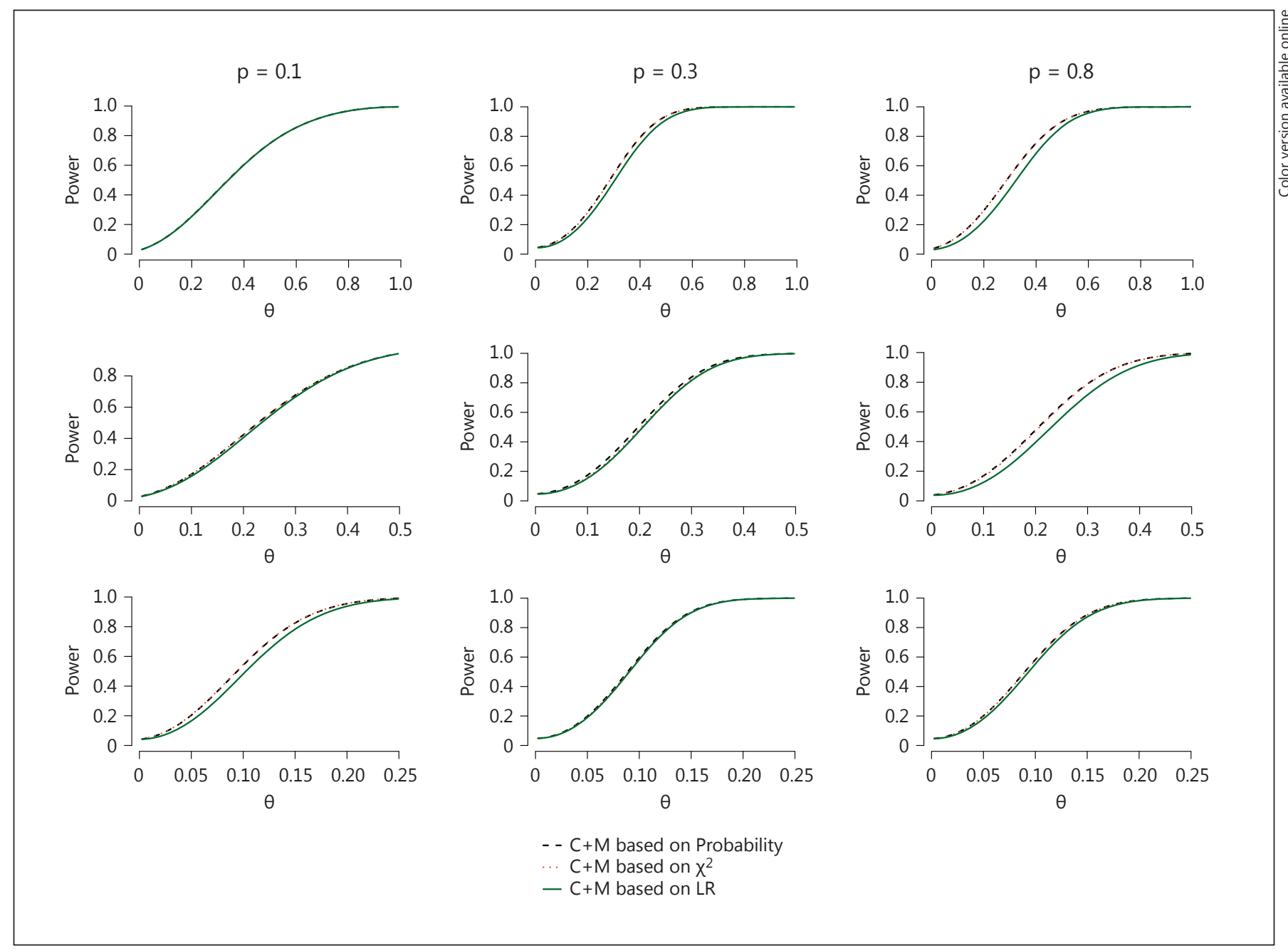

Fig. 4. Power comparison for the $\mathrm{C}+\mathrm{M}$ approach based on the three different test statistics at the nominal level of 0.05 for $N=50,100$, and 500 in the rows 1,2 , and 3, respectively.

The C+M approach has been successfully applied to test the difference between two binomial proportions [26] and the independence of exchangeable binary data with equal-sized clusters [17]. This unconditional approach has been recommended for use in practice due to its efficiency relative to other testing procedures [26].

\section{Results}

To evaluate the performance of the three exact testing procedures (the $\mathrm{C}$ approach, the $\mathrm{M}$ approach, and the $\mathrm{C}+\mathrm{M}$ approach), the exact type I error rates were compared at the nominal significance levels $\alpha=0.01$ and $\alpha=$ 0.05 . Figure 1 shows the type I error rates of the tests as a function of the true value of the nuisance parameter with a total sample size $N=20,80,200$, and 1,000. Exact conditional and unconditional tests respect the test size, so the maximum of each curve in the figure is less than or equal to the nominal level. As seen in figure 1, the $\mathrm{C}$ approach and the $\mathrm{M}$ approach are conservative for small to medium sample sizes. The $\mathrm{M}$ approach is very conservative for the majority of $\mathrm{p}$ values with the actual type I error rate far below the nominal level. The curve of the $\mathrm{C}+\mathrm{M}$ approach is much closer to the nominal level than those of the other two exact approaches. The difference between the $\mathrm{C}$ approach and the $\mathrm{C}+\mathrm{M}$ approach becomes small for large sample sizes. 
When the HWE does not hold, a second parameter is needed to fully define the probabilities of the three diallelic genotypes $A A, A B$, and $B B$. One example [16] is

$$
\begin{aligned}
& p_{A A}=p^{2}+\theta p(1-p), \\
& p_{A B}=2 p(1-p)(1-\theta), \\
& p_{B B}=(1-p)^{2}+\theta p(1-p),
\end{aligned}
$$

where $\theta$ is the inbreeding coefficient [27] and $\theta \in[\max$ $(-p /(1-p),-(1-p) / p), 1]$. When $\theta=0$, the probabilities satisfy HWE. We first compare the $\mathrm{C}$ approach, the $\mathrm{M}$ approach, and the $\mathrm{C}+\mathrm{M}$ approach based on the $T_{\chi}{ }^{2}$ in figure 2 for $p=0.1,0.2$, and 0.7 , respectively. The power plot is drawn as a function of $\theta$ for each given $p$. As seen in the figure, the power is an increasing function of $\theta$ for $\theta \in[0,1]$. For the configurations considered, the $C+M$ approach is generally more powerful than the other two exact tests. For small sample sizes, the $\mathrm{M}$ approach has higher power than the $\mathrm{C}$ approach, which confirms the results found by Haber [16]. The M approach is less powerful than the C approach for large sample sizes. Similar results were observed for the comparison based on $T_{L R}$ and $T_{\text {Probability. The }} \mathrm{C}+\mathrm{M}$ approach is therefore recommended due to the power gain.

We now compare the $\mathrm{C}+\mathrm{M}$ approach based on $T_{\text {Probability }}, T_{\chi^{2}}$, and $T_{L R}$ with respect to its power. The power of the $\mathrm{C}+\mathrm{M}$ approach using different test statistics with $p=0.1,0.3$, and 0.8 is shown in figure 3 for $\alpha=0.01$ and in figure 4 for $\alpha=0.05$. As seen in both figures, the difference in the power of the $\mathrm{C}+\mathrm{M}$ approach based on $T_{\text {Probability }}$ and the $\mathrm{C}+\mathrm{M}$ approach based on $T_{\chi}{ }^{2}$ is negligible. Although the $\mathrm{C}+\mathrm{M}$ approach based on $T_{L R}$ is slightly more powerful than the $\mathrm{C}+\mathrm{M}$ approach based on the other two test statistics for small $\mathrm{p}$ values and small sample sizes, it is generally less powerful than the other two. We would recommend to use the $\mathrm{C}+\mathrm{M}$ approach based on $T_{\text {Probability }}$ or $T_{\chi^{2}}$.

\section{Discussion}

The computation time for the $\mathrm{C}+\mathrm{M} \mathrm{p}$ value is longer than those for the $C \mathrm{p}$ value and the $\mathrm{M}$ p value because of the larger sample space for the $\mathrm{M}$ approach. For example, for a sample size of 100 , the genotype frequencies $b f n=\left(n_{A A}, n_{A B}, n_{B B}\right)=(75,15,10)$. The total number of sample points in the sample space is $102 \times 101 / 2=5,151$ for the $\mathrm{M}$ approach, while there are only 36 for the $\mathrm{C}$ approach given the number of $B$ genes $\left(n_{B}=35\right)$. This may be the reason why the $C$ approach is more conservative than the unconditional approaches. This may also be the reason why the unconditional approach is more computational than the $\mathrm{C}$ approach. We have made our effort to improve the algorithm for the unconditional approach, and it only takes $15 \mathrm{~s}$ to find the actual type I error rate of the $\mathrm{C}+\mathrm{M}$ approach at the significance level of $\alpha=0.05$ for sample sizes up to $N=1,000$ on personal computers. We are working on an $\mathrm{R}$ package to implement the $\mathrm{C}$ approach, the $\mathrm{M}$ approach, and the $\mathrm{C}+\mathrm{M}$ approach based on the three test statistics considered in this study. The source code is available from the first author upon request. In this study, we compared the exact testing procedures to loci with two alleles. In the case of multiple alleles, there are multiple nuisance parameters in the null likelihood. It is still a computational challenge to find the maximum of the null likelihood with multiple nuisance parameters.

Although we investigated the unconditional approaches based on three commonly used test statistics, other test statistics may also be considered, such as the Bayes factor $[5,28]$. The extension of the unconditional approaches using other test statistics is easy as it would be very similar to the testing procedure described in this study. An alternative for testing the HWE is the Bayesian method (see Wakefield [29] and Consonni et al. [30]). Priors in the Bayesian method were investigated and suggested by Consonni et al. [30]. The comparison between the Bayesian method and the $\mathrm{C}+\mathrm{M}$ approach would be an interesting topic, and we consider this as future work.

\section{Acknowledgement}

The author would like to thank two referees' comments to improve the manuscript.

References population. Science 1908;28:49-50.

$\checkmark 2$ Crow JF: Eighty years ago: the beginnings of population genetics. Genetics 1988;119:473476.

3 Edwards AW: G.H. Hardy (1908) and HardyWeinberg equilibrium. Genetics 2008;179: $1143-1150$

4 Chakraborty R, Zhong Y: Statistical power of an exact test of Hardy-Weinberg proportions of genotypic data at a multiallelic locus. Hum Hered 1994;44:1-9.

5 Montoya-Delgado LE, Irony TZ, de B Pereira CA, Whittle MR: An unconditional exact test for the Hardy-Weinberg equilibrium law: sample-space ordering using the Bayes factor. Genetics 2001;158:875-883. 
6 Kang SH, Shin D: The size of the chi-square test for the Hardy-Weinberg law. Hum Hered 2004;58:10-17.

7 Engels WR: Exact tests for Hardy-Weinberg proportions. Genetics 2009;183:1431-1441.

$>8$ Elston RC, Forthofer R: Testing for HardyWeinberg equilibrium in small samples. Biometrics 1977;33:536-542.

9 Weir BS: Genetic Data Analysis II: Methods for Discrete Population Genetic Data, ed 2. Sunderland, Sinauer Associates, Inc., 1996.

10 Wigginton JE, Cutler DJ, Abecasis GR: A note on exact tests of Hardy-Weinberg equilibrium. Am J Hum Genet 2005;76:887-893.

11 Guo SW, Thompson EA: Performing the exact test of Hardy-Weinberg proportion for multiple alleles. Biometrics 1992;48:361-372.

-12 Mehta CR, Patel NR: A network algorithm for performing Fisher's exact test in $\mathrm{r}$ by c contingency tables. J Am Stat Assoc 1983;78:427434.

13 Barnard GA: A new test for $2 \times 2$ tables. Nature 1945;156:177.

14 Basu D: On the elimination of nuisance parameters. J Am Stat Assoc 1977;72:355-366.

15 Shan G, Ma C, Hutson AD, Wilding GE: An efficient and exact approach for detecting trends with binary endpoints. Stat Med 2012; $31: 155-164$
16 Haber M: An exact unconditional test for the Hardy-Weinberg equilibrium. Biom J 1994; 36:741-749.

17 Shan G: More efficient unconditional tests for exchangeable binary data with equal cluster sizes. Stat Probab Lett 2013;83:644-649.

18 Irony TZ, Pereira CA: Exact tests for equality of two proportions: Fisher v. Bayes. J Stat Comput Simul 1986;25:93-114.

19 Pereira CA, Lindley DV: Examples questioning the use of partial likelihood. J R Stat Soc Series D (The Statistician) 1987;36:15-20.

20 Maiste PJ, Weir BS: A comparison of tests for independence in the FBI RFLP data bases. Genetica 1995;96:125-138.

21 Rohlfs RV, Weir BS: Distributions of HardyWeinberg equilibrium test statistics. Genetics 2008;180:1609-1616.

22 Brentani H, Nakano EY, Martins CB, Izbicki R, Pereira CA: Disequilibrium coefficient: a Bayesian perspective. Stat Appl Genet Mol Biol 2011; 10:article 22.

23 Shan G, Ma C: Unconditional tests for comparing two ordered multinomials. Stat Methods Med Res 2012, E-pub ahead of print.
24 Shan G, Ma C, Hutson AD, Wilding GE: Some tests for detecting trends based on the modified Baumgartner-Weiss-Schindler statistics. Comp Stat Data Anal 2013;57:246261.

25 Boschloo RD: Raised conditional level of significance for the $2 \times 2$-table when testing the equality of two probabilities. Stat Neerl 1970;24:1-9.

26 Berger RL: Power comparison of exact unconditional tests for comparing two binomial proportions. Technical report, Department of Statistics, North Carolina State University, 1994.

27 Emigh TH: A comparison of tests for HardyWeinberg equilibrium. Biometrics 1980;36: 627-642.

-28 Pereira CA, Nakano F, Stern JM, Whittle MR: Genuine Bayesian multiallelic significance test for the Hardy-Weinberg equilibrium law. Genet Mol Res 2006;5:619-631.

29 Wakefield J: Bayesian methods for examining Hardy-Weinberg equilibrium. Biometrics 2010;66:257-265.

30 Consonni G, Moreno E, Venturini S: Testing Hardy-Weinberg equilibrium: an objective Bayesian analysis. Stat Med 2011;30:62-74. 